The Arbutus Review • Fall 2015 • Vol. 6, No. 1

\title{
Une analyse de l'intertextualité dans Paul a un travail d'été par Michel Rabagliati
}

\author{
Brynn Fader * \\ The University of Victoria \\ brynnfader@alumni.ubc.ca
}

\begin{abstract}
Résumé
Cet article étudie les références implicites et explicites au Petit Prince qui figurent dans Paul a un travail d'été de Michel Rabagliati. La différence entre le comportement des enfants et celui des adultes autour d'eux qui suscite le découragement, de même que les thèmes de la transformation grâce à l'amitié et du sens des responsabilités, sont traités en des termes provenant du texte de Saint-Exupéry. Les références au thème d'un adolescent qui mature à travers la vie permettent au public de Rabagliati de se retrouver dans sa bande dessinée, car les expériences de Paul résonnent avec celles des lecteurs.
\end{abstract}

Les mots clés : Bande dessinée; coprésence; explicite; implicite; intertextualité; références

$\coprod$ e lecteur attentif de Paul a un travail d'été de Michel Rabagliati repère de nombreuses références au Petit Prince dans ce roman graphique. Ces rapports sont de plusieurs natures et constituent des références intertextuelles, tantôt implicites, tantôt explicites : la différence entre le comportement des enfants et celui des adultes autour d'eux qui suscite le découragement, la transformation grâce à l'amitié, la notion de responsabilité, le symbole de l'étoile et la vision qui vient du c. Je vais les aborder en ordre chronologique de lecture et de découverte, de manière à ce que les allusions explicites soient discutées en premier et les allusions muettes ensuite. Cet essai va prouver que les leçons du Petit Prince sont toujours actuelles. Rabagliati est donc un exemple contemporain qui utilise des références intertextuelles aux leçons du Petit Prince dans sa bande dessinée. Il le fait en situant l'histoire au Québec, avec les personnages réalistes, les premières amours, les camps d'été, les enfants et les familles. Il crée une atmosphère où le potentiel de maturation pour un adolescent à l'âge adulte se produit. Autrement dit, il décrit un monde où un adolescent grandit avec les leçons tirées de l'histoire du Petit Prince.

Selon Gérard Genette, spécialiste de la littérature, «l'intertextualité »(8) est une relation définit comme une coprésence, souvent identifiée dans «[s]a forme la plus explicite et la plus littérale »(8) c'est-à-dire par «la pratique traditionnelle de la citation (avec guillemets, avec ou sans référence précise) »(8). En fait, c'est la présence «d'un texte dans un autre »(8). Paul a un travail d'été fait référence au Petit Prince et ces deux textes créent donc une coprésence. En mentionnant Le Petit Prince dans sa bande dessinée, Rabagliati crée «une relation de coprésence »(Genette, 8) entre deux textes.

Le Petit Prince est une œuvre pour les enfants traduite en plus de 200 langues. C'est l'un des livres les plus populaires dans le monde. Le narrateur est un pilote de l'air, victime d'un écrasement dans le Sahara. C'est là où le narrateur rencontre le Petit Prince qui s'occupe d'une image d'un mouton. Le Petit Prince explique qu'il est venu de l'astéroïde B 612, qu'il a quitté car sa fleur lui a

\footnotetext{
${ }^{*}$ Je tiens à remercier mon professeur et mon mentor, Mme. Hélène Cazes qui est une source d'encouragements et des conseils sans fin. De plus, je tiens à remercier ma famille d'avoir toujours été en faveur de mes objectifs
} 
menti. Le Petit Prince est déterminé à rencontrer de nouveaux amis. Il rencontre plusieurs hommes étranges, un renard et un serpent, chacun lui enseignant une leçon de responsabilité, de vie, d'amour ou d'apprivoisement.

Paul a un travail d'été est une bande dessinée québécoise qui raconte la vie d'un homme à travers sa maturation. Paul est déçu de sa vie jusqu'à ce qu'il rencontre Annie et les campeurs à un camp d'été où il obtient un emploi d'été comme moniteur. Grâce à Annie, Paul apprend la responsabilité ainsi que l'apprivoisement et il trouve l'amour. Il comprend qu'il faut avoir ces telles choses dans le but d'avoir une vie heureuse; sans quoi l'on devient tellement triste. Plus tard de sa vie, il exprime ces leçons à sa fille qui en comprend bien l'importance.

La première allusion explicite au Petit Prince qui se trouve dans la bande dessinée est celle de l'étoile. Les étoiles sont un symbole et représentent l'amour éternel. Par exemple, dans Paul a un travail d'été, l'étoile est d'abord un symbole de l'amour éternel entre Annie et Paul. Annie lui donne un cadeau qui est placé dans le ciel à perpétuité : «c'est un cadeau que tu ne peux pas prendre dans tes mains et qui est très loin d'ici! hi hi! »(129). Ce cadeau est une représentation symbolique de la relation entre Annie et Paul qui peut être trouvée n'importe où. L'étoile figure dans Le Petit Prince à la page 79 , où le renard dit :

Tu regarderas, la nuit, les étoiles. C'est trop petit chez moi pour que je te montre où se trouve la mienne...mon étoile, ça sera pour toi une des étoiles. Alors, toutes les étoiles, tu aimeras les regarder. . elles seront toutes tes amies. Et puis, je vais te faire un cadeau.

La relation significative est symbolisée par cette étoile, visible de partout. De la même manière, Annie et Paul seront toujours ensembles, jamais seuls. Paul a confié ce secret, la référence au Petit Prince, à sa fille curieuse et fascinée. Il lui montre l'emplacement de la planète $\mathrm{B} 612$, pour laquelle il indique une étoile dans le ciel. «Tout juste au-dessus de la deuxième étoile, collée, collée dessus, il y en a une toute petite que personne ne remarque "(Rabagliati, 151). Il a choisi une étoile seulement pour elle. Cette action est semblable à celle d'Annie, qui avait donné, des années plus tôt, l'emplacement d'une étoile à Paul.

Dans Paul a un travail d'été, l'étoile représente un symbole d'amour dans le ciel, où que sa fille se trouve, à tout moment de sa vie. L'amour est invisible et devient plus beau grâce à son invisibilité même. Ce concept d'invisibilité est tiré de la citation du Petit Prince : ce qui est essentiel est «invisible pour les yeux »(Saint-Exupéry, 65). Dans ce cas, c'est l'amour entre Paul et sa fille qui est invisible. En outre, le renard attire l'attention sur le fait que l'étoile est très belle parce qu'elle est unique (on peut ajouter que cela est semblable à la fleur, qui elle aussi est unique) et qu'elle lui appartient. Le renard en fait cadeau au Petit Prince. De la même manière, la fille de Paul peut voir éternellement que l'étoile de son père lui est unique. Quand Annie a fait ce cadeau significatif à Paul, il a pleuré. Grâce à ces larmes, on comprend que ce cadeau a beaucoup de mérite et qu'il aime beaucoup Annie, bien que certainement d'un amour différent de celui qu'il porte à sa fille Alice. Cette étoile représente ainsi deux types d'amour : l'amour entre une fille et son père et l'amour entre deux amants.

Pour illustrer cela, sa fille portera l'étoile partout avec elle. Comme le Petit Prince et le renard, Paul et sa fille sont devenus uniques l'un pour l'autre et sa fille pensera au Petit Prince. Paul avait dessiné une image à l'école secondaire du Petit Prince et du renard (Rabagliati, 12). À l'âge adulte, son intérêt pour Le Petit Prince a continué à se manifester lorsqu'il a donné l'étoile à sa fille.

L'allusion explicite centrale, voir avec le cœur, est illustrée par deux exemples. Le premier est que Paul applique indirectement cette maxime en s'occupant de Marie, la petite fille aveugle qui séjourne au camp où il travaille. Il apprend, tout au long du temps qu'il passe avec elle, qu'elle est gentille, douce et heureuse malgré son handicap; elle refuse d'abandonner (Lloyd, 54). Paul, qui a adopté les comportements d'un adulte, a besoin de comprendre comment «voir avec le cœur »pour 
trouver le bonheur malgré les difficultés qui peuvent survenir. On peut conclure que les enfants dans ce roman graphique voient naturellement avec le cœur. Plus profondément, l'esprit d'un enfant est par nature plus optimiste et les enfants sont occupés par l'imagination et la rêverie (Hughes et King, 68). Un exemple de cela est Marie, de même que la fille de Paul, car elles expriment qu'il faut toujours voir avec le cœur, en dépit du mal. L'esprit ouvert de Marie est personnifié par la poupée que Paul, adulte, trouvera sur le terrain abandonné. Cette poupée est ensuite donnée à sa fille, qui fait référence à l'œuvre dans un dialogue avec son père. Elle remarque immédiatement : «heille! elle n'a pas d'yeux! »(Rabagliati, 150). Ceci pousse Paul à dire «qu'elle est comme le Petit Prince; elle n'a pas besoin d'yeux pour voir, elle voit avec son cœur...»(150). La poupée présente un trait physique de Marie, la cécité. Avant d'avoir trouvé la poupée, à l'âge adulte, Paul avait besoin d'un souvenir de Marie et du camp, n'arrivant pas à être en paix avec lui-même. La poupée qu'elle a laissée au camp des années auparavant représente et perpétue le souvenir de Marie. Ensuite, c'est la fille de Paul qui lui rappelle de voir avec son cœur lorsqu'il est adulte. Alex Farris, journaliste, traduit que ce qui est essentiel dans la vie est presque toujours invisible à l'œil nu (par. 2). Cela est évident dans l'exemple de Paul qui se souvient de Marie dès qu'il trouve cette poupée aveugle. C'est donc Marie qui provoque ce sentiment de voir avec le c œur.

Une leçon du Petit Prince est le secret essentiel du renard : «on ne voit bien qu'avec le cœur. L'essentiel est invisible pour les yeux »(Saint-Exupéry, 78). Rabagliati incorpore cette leçon dans sa bande dessinée. Le renard explique que ce secret est «habituellement oublié »(Galembert, 22) et toujours oublié par les adultes. Rabagliati démontre que ce secret est oublié par Paul lui-même. Cette phrase a été utilisée dans Paul a un travail d'été dans le but d'expliquer le message positif : on n'a pas besoin des yeux pour voir la beauté de la vie ou du monde, une vérité répétée par Marie. Cette notion vient du Petit Prince : «les yeux sont aveugles. Il faut chercher avec le cœur »(Saint-Exupéry, 73). En fait, Marie a besoin de chercher avec son c our, car elle est aveugle. Cet esprit positif est celui auquel Paul s'adapte pendant ses vacances au camp. Dans Le Petit Prince, «les étoiles et la fleur sont des images du poète (Saint-Exupéry) [qui aident le Prince à devenir émotionnellement] ouvert aux autres et au monde »(Pagé, 96). C'est la fleur qui aide le Petit Prince à devenir plus ouvert et c'est Marie qui aide Paul. L'idée «de voir avec le cœur »est une leçon de vie essentielle tirée du conte de Saint-Exupéry, et nous voyons à travers la bande dessinée que cette leçon est transmise de génération en génération.

Ayant comparativement discuté les références explicites, on doit donc exposer les références implicites. La première allusion est la différence du comportement entre les adultes et les adolescents, laquelle crée une tension entre eux. Dans Paul a un travail d'été, Paul a l'impression que les adultes ne le comprennent pas, car le directeur ne lui permet pas de terminer son projet d'une fresque du Petit Prince et du renard. Paul est très passionné et a «envie de faire ce projet-là plus que tout au monde...ça [lui] tient vraiment à cœur »(Rabagliati, 14). Cependant, il semble raisonnable au directeur de ne pas lui permettre de le réaliser à cause de ses mauvaises notes scolaires. En dépit du fait que la décision semble raisonnable, l'expression de sourire sur le visage du directeur est insensible, distante et impassible. La réaction du directeur et sa décision d'empêcher Paul de travailler davantage sur le projet illustrent que les adultes ont des priorités différentes de celles des enfants. Tandis que le directeur pense que les notes sont plus importantes que la fresque, Paul est de l'avis contraire. Néanmoins, Paul est devenu extrêmement déçu et perturbé à cause du directeur, car celui-ci n'exprime pas de compassion. Or, la fresque irréalisée devient l'album Paul a un travail d'été, une fresque inspirée par Le Petit Prince, retravaillée et mise en narration.

Pareillement, le narrateur dans Le Petit Prince est déçu parce que les adultes ne comprennent pas le dessin du serpent qui digère un éléphant. Les adultes interprètent le dessin de manière littérale et voient seulement un chapeau; il manque aux adultes la capacité de se servir de leur imagination (Saint-Exupéry, 57), et donc l'aptitude de voir la vraie image d'un serpent qui mange un éléphant. 
Le narrateur déclare avoir eu «au cours de [sa] vie des tas de contacts avec des tas de gens sérieux. [Il a] beaucoup vécu chez les grandes personnes »(Saint-Exupéry, 3). Par conséquent, on voit un parallèle dans un comportement différent entre les adultes et les enfants à partir des deux textes. Cette notion est importante parce qu'elle établit une différence fondamentale entre les deux groupes et est décrite comme le problème initial pour Paul et le Petit Prince.

Plus profondément, la question est de savoir pourquoi ces deux groupes ne se comprennent pas. On en vient à la conclusion que les adultes et les enfants se préoccupent de choses différentes. D'un côté,

[Les adultes sont] piégés par le côté matériel, vulgaire de l'existence, victimes de leur vanité, de leur cupidité ou de leur paresse intellectuelle, les «grandes personnes »jugent le propos de quelqu'un d'après son costume [c'est le cas de l'astronome turc], évaluent la beauté d'une maison d'après son prix et croient connaître un jeune ami d'après les revenus de son père (Hughes et King, 68).

Le narrateur développe cette idée en disant qu'il «ne lui parlai[t] ni de serpents boas? ni d'étoiles.... [Il] lui parlai[t] de bridge, de golf, de politique et de cravates »(Saint-Exupéry, 3). Les adultes s'occupent par les «jeux fonctionnels basés sur la répétition (l'homme vaniteux), jeux fictionnels esquissés avec le roi et menés à terme avec le renard (jouer à rendre la justice) et jeux d'acquisition exerçant la mémoire »(Biagioli, 33) ou «acquièrent [seulement] des connaissances stables sur l'astronomie, le système solaire et le comput du temps »(33). Ils sont complètement consommés par les activités quotidiennes; tellement absorbés, en fait, qu'ils n'ont pas de relations avec les autres et sont seuls. Leurs activités divergent de celles des enfants, car le monde adulte est si compliqué (Manoll, 20). De l'autre côté, les enfants s'occupent «de jouer, de [rêver]»(68), d'imaginer, d'aimer sans réserve, veulent un ami et ont le «besoin de communiquer avec quelqu'un »(Pagé, 89). Par exemple, ce trait se retrouve chez la jeune fille de Paul et chez le Petit Prince. Il veut un ami, tandis qu'elle imagine et aime sans réserve. Le Petit Prince déclare «je suis responsable de ma rose...»(33) et la fille de Paul a hâte de parler du Petit Prince, de chercher la planète B 612 dans le ciel et de parler à son père. Les adultes et les enfants sont tellement différents parce que leurs comportements et leurs priorités diffèrent. Il n'est donc pas surprenant qu'ils soient souvent en opposition.

Une autre référence intertextuelle traite du comportement : elle se trouve lorsque l'adolescent Paul démontre les mêmes tendances que les adultes et contre lesquelles le renard met en garde le Petit Prince. Par conséquent, Paul ne peut construire de vraies amitiés que lorsqu'il abandonne son travail à l'imprimerie et la maison de ses parents pour se rendre au camp : il doit s'y faire des amis dans le but de survivre en tant que moniteur et doit prendre soin d'autres personnes que lui-même. Avant cela, Paul était seul. C'est précisément ce que dit le renard au Petit Prince : en tant qu'adulte, vous ne pouvez ni oublier vos amitiés, ni vous adapter au comportement majoritaire, et ni non plus oublier la force et les devoirs de l'apprivoisement. La leçon d'apprivoisement suggère que si tu veux un ami, il faut l'apprivoiser dans le but de rendre cet ami unique. Cette allusion ne lie pas de fait Paul et le comportement du Petit Prince, mais lie plutôt le comportement des adultes aux attitudes contre lesquelles le renard met son ami en garde; ainsi, la transformation que Paul a subie est adaptée à celle que le renard juge un comportement approprié (Monin, 171).

La transformation de Paul grâce à l'amitié et à l'amour se lit également par allusion au Petit Prince. Paul a établi des amitiés avec Annie et Marie qui l'aident à progresser. Par exemple, il craint pour l'enfant qui a failli se blesser en canoë (117), il aide Marie à imaginer le paysage en le lui décrivant (101), il l'aide à chercher la poupée qu'elle a perdue et puis, quand elle n'a pas pu la trouver (114), il dort à côté d'elle (115). Il encourage aussi un autre campeur à la conquête de son vertige alors que le groupe fait de l'escalade (74). En devenant moniteur du camp avec Annie, il se dispute avec elle lorsqu'elle lui reproche d'avoir peu de patience et de compassion (70). Sa collègue 
Annie le fait réfléchir sur sa propre vie et il se rend compte qu'il a une vie facile (70-1), surtout en comparaison avec les campeurs. Elle l'a donc aidé à progresser dans son comportement parce qu'elle le critique (77) et il a changé son point de vue sur la vie. Il ne pense plus qu'il est malheureux et il est devenu reconnaissant de son sort. De plus, parce que la petite Marie est aveugle, il lui est nécessaire de changer afin de prendre soin d'elle aussi bien que des autres campeurs et ainsi réussir dans son travail au camp. Mais c'est grâce à Annie et Marie, qu'il mûrit. Avant la transformation, Paul regrettait d'avoir pris le poste de conseiller.

Les choses changent avec l'arrivée d'une campeuse aveugle qui refuse de se rendre à son handicap. Leur liaison permet à Paul découvrir un sens de soi, l'ouverture aux gens et le monde autour de lui. Vingt ans plus tard, Paul semble en colère et cynique. Quand il revient, son cœur s'ouvre à nouveau, l'attirant à sa fille dans un point culminant de catharsis émotionnelle (Lloyd, 54).

On voit la progression de Paul lorsque le jeune campeur dit que «c'est l'fun avec toi »(Rabagliati, 75 ) et quand il se rend compte que les campeurs l'aiment, «euh...p...pis moi aussi j'aime ça avec vous autres, les gars...»(75). La progression est bien marquée par le fait qu'il commence à accepter ses émotions durant le départ de son premier groupe de campeurs et de celui de Marie.

En particulier, l'amour entre Paul et Annie déclenche aussi la progression de Paul. Leur relation amoureuse devient évidente quand Paul est fasciné par le corps d'Annie et quand il se dispute avec elle (une préfiguration définitive de l'amour) (Lloyd, 54). Grâce à elle et à leur relation, il apprend comment s'abstenir de hurler et comment aider les autres. Lorsqu'il adopte les traits comportementaux d'un adulte, il n'est pas mûr. Il éprouvera les premières «douleurs de l'amour...confrontera des échecs, et le sentiment d'être rejeté ou accepté »(Hughes et King, 68). Il n'adopte plus les comportements d'un adolescent, tels que d'être content, imaginatif et d'avoir un esprit ouvert, ou de faire preuve d'amour sans condition. Malgré les traits qu'il possède au début de la bande dessinée, Paul démontre sa capacité à changer grâce aux expériences qu'il vit au camp, à sa relation avec Annie et en s'adaptant à sa mission de chef en collaboration avec elle.

De même que Paul, le Petit Prince a progressé grâce à l'amitié et l'amour avec la fleur, mais c'est le renard qui l'aide à accepter ses sentiments pour la fleur, pour qu'il puisse l'aimer telle qu'elle est. D'une certaine façon, le renard est Marie, comme la fleur est Annie pour Paul. Bien qu'il soit difficile pour le Petit Prince d'avoir une relation réciproque avec la fleur, il a besoin de cette relation. La relation avec elle se relie au message du renard parce que celui-ci prône la loyauté et le dévouement au Petit Prince. En dépit de la multiplicité des fleurs, l'amour pour une seule fleur dépasse la pluralité des amours possibles. Il explique aussi qu'il a déjà apprivoisé sa fleur car il en prend soin. Plus spécifiquement, le Petit Prince ń sera pour [le renard] unique au monde (Saint-Exupéry, 61). L'amitié avec la fleur conduit le Petit Prince vers un voyage, en raison du mensonge de la fleur, au cours duquel il apprend à aimer inconditionnellement et le véritable besoin et sens de l'amitié. Cette progression est marquée par le fait qu'il a changé d'opinion à propos de la fleur. Après il se rend compte du fait que c'est à lui de s'occuper de ses responsabilités (des campeurs, d'Annie, de Marie, de lui-même). Il a mûri émotionnellement.

Le premier amour d'un adolescent se produit souvent au cours d'un point de maturation particulier ou pendant "une période initiatique" (Paquin, 4). C'est le cas pour Paul. Son premier amour se produit pendant la maturation hormonale. Plus précisément, Paul a commencé à s'intéresser au sexe, aux filles et à l'amour, notamment après avoir rencontré Annie au camp (Rabagliati, 18, 127). En même temps, la relation entre Paul et Annie montre que Paul grandit grâce à l'apprivoisement d'une relation importante et qu'il a internalisé des leçons du Petit Prince.

Au cours de son adolescence, Paul est chargé du bien-être des campeurs; il est responsable de quelqu'un d'autre que lui-même. Plus tard, au cours de sa vie d'adulte, il sera responsable de sa 
The Arbutus Review • Fall $2015 \bullet$ Vol. 6, No. 1

petite fille. La responsabilité est ainsi un autre exemple implicite. Par conséquent, tout au long de ce roman graphique, Paul illustre son sens des responsabilités par le soin des autres. Autrement dit, Paul a une obligation envers sa fille en tant que père, et comme chef de camp, envers les campeurs, notamment Marie. Le fait qu'il a rencontré Marie au cours de son adolescence, qu'il l'aime et qu'il se soucie d'elle, l'aidera à apprendre le sens des responsabilités et c'est une leçon qui influencera sa relation avec sa fille.

En revanche, le Petit Prince était en colère contre la fleur parce qu'elle a menti; il est assez fâché pour quitter sa planète qu'il avait également prise en charge. Il est responsable du bien-être de sa fleur et de sa planète. Le renard a enseigné au Prince à «répondre de ses actions? [c'est] une connaissance difficile à acquérir...»(Monin, 162). D'autres exemples illustrent cette même notion de sa capacité d'être responsabilisé : celui des baobabs qui ont besoin d'être élagués (Saint-Exupéry, 15) et celui de la fleur qui a besoin également d'être protégée (24-7). Pour le dire autrement, pendant son voyage et en répondant au renard et aux hommes sur les planètes, le Petit Prince prend conscience de ses responsabilités envers sa planète ainsi que la fleur. Plus profondément, le Petit Prince et la Rose, désirent être aimés (Monin, 146). La fleur (pour le Prince) et Marie (pour Paul) sont donc les «catalyseurs uniques »(162) qui conduisent la responsabilité de Paul et du Petit Prince, ce qui est un indicateur (d'un type) d'amour.

En général, on voit que Paul doit apprendre, avec difficulté, le sens des responsabilités dans des situations difficiles. D'après les notes de l'auteur, son «vrai »travail, le camp d'été, et se responsabiliser n'ont pas été faciles pour lui. Cela est le cas pour Paul, de même que pour de nombreux adolescents actuellement. En présentant honnêtement ces difficultés, Rabagliati partage avec le monde les difficultés qu'un adolescent peut rencontrer avant de prendre ses responsabilités, avec tous les sacrifices et les luttes qui accompagnent ce mûrissement. Il y a là un parallèle avec le Petit Prince, qui a du mal à assumer sa responsabilité envers la fleur mais qui est aisément à la hauteur de la tâche pour le bien-être de la planète. Par conséquent, on peut dire que Rabagliati nous apprend par sa fiction qu'il est difficile, même dans une histoire inventée, de reconnaître ses responsabilités. Rabagliati met en œuvre ce concept en le combinant avec les leçons du Petit Prince et en les projetant dans Paul, qui est un personnage plus réaliste et actuel que le Petit Prince. Paul a un travail d'été est donc un «travail modeste ... [qui prend] conscience de la confiance et de l'affection $»(4)$. La bande dessinée est un voyage à travers l'adolescence dans lequel beaucoup peuvent se retrouver pour dire que les leçons du Petit Prince sont toujours actuelles. Paul a un travail d'été fait usage d'allusions intertextuelles à la fois explicites et implicites qui tirent leur origine du livre Le Petit Prince.

\section{RÉfÉREnCES BibliogRAPHiques}

Biagioli, N. (2001). Le dialogue avec l'enfance dans Le Petit Prince. Études Littéraires 33 :2, 27-42. www.ebscohost.com http://dx.doi.org/10.7202/501291ar

Brunet, Martine (2013). Les Pauls de Michel Rabagliati. Québec français, 168, 108. www.érudit.com

Farris, Alex (2011, 26 juilliet). «Book Review : The Little Prince.»www.yahoo.com

Galembert, Laurent de (2002, 26 novembre). La grandeur du Petit Prince. Le Manuscrit, 1-98. http ://nitescence.free.fr/DEA.pdf

Gérard, Genette (1982). Palimpsestes. Paris : Seuil. 
Hughes, Janette, et Alyson E. King (2010). Dual Pathways to Expression and Understanding : Canadian Coming-of-Age Graphic Novels. Children's Literature in Education, 68-73. www.ebscohost.com http://dx.doi.org/10.1007/s10583-009-9098-8

Lloyd, Chelsey (2003). 'Paul Has a Summer Job.Broken Pencil, 22, 54.

Manoll, Michel (1961). Saint-Exupéry, Prince des Pilotes. Paris : Société Nouvelle des Éditions G.P.

Monin, Yves (1976). L’Ésotérisme du Petit Prince de Saint-Exupéry. Paris : Éditions A.-G. NIZET.

Noel-Gaudreault, Monique (2013). Comment Michel Rabagliati a écrit certains de ses livres. Québec français, 168, 106-107. www.érudit.com

Pagé, Pierre (1963). Saint-Exupéry et le Monde de L'Enfance. Montréal : Fides.

Paquin, Eric (2005, mars-avr.) Souvenirs de jeunesse. Spirale Magazine. www.érudit.com

Rabagliati, Michel (2003). Paul a un travail d'été. (Helge Dascher, Trans.). Montréal : Drawn and Quarterly.

Saint-Exupéry, Antoine de (1943). Le Petit Prince. Paris : Harcourt, Inc.

Viau, Michel (2008). La BD au Québec : une route semée d'embûches. Québec français 149, 32-34. 